\title{
The Use of Injectable Chitosan/Nanohydroxyapatite/Collagen Composites with Bone Marrow Mesenchymal Stem Cells to Promote Ectopic Bone Formation In Vivo
}

\author{
Bo Yu, ${ }^{1}$ Yichen Zhang, ${ }^{2}$ Xiaoming Li, ${ }^{3}$ Qiongren Wang, ${ }^{2}$ Yi Ouyang, ${ }^{2}$ Yuanyou Xia, \\ Bo Yu, ${ }^{2}$ Bomiao Lin, ${ }^{4}$ Songjian Li, ${ }^{1}$ Yubo Fan, ${ }^{3}$ and Yan Chen ${ }^{5}$ \\ ${ }^{1}$ Department of Orthopedics, Zhujiang Hospital of Southern Medical University, Guangzhou 510282, China \\ ${ }^{2}$ The Second Clinical Medical College of Southern Medical University, Guangzhou 510282, China \\ ${ }^{3}$ Key Laboratory for Biomechanics and Mechanobiology of Ministry of Education, School of Biological Science and Medical Engineering, \\ Beihang University, Beijing 100191, China \\ ${ }^{4}$ Department of Radiology, Zhujiang Hospital of Southern Medical University, Guangzhou 510282, China \\ ${ }^{5}$ Department of Ultrasonic Diagnosis, Zhujiang Hospital of Southern Medical University, Guangzhou 510282, China
}

Correspondence should be addressed to Xiaoming Li; x.m.li@hotmail.com and Yan Chen; smu_chen@163.com

Received 13 June 2013; Accepted 14 August 2013

Academic Editor: Shuming Zhang

Copyright (C) 2013 Bo Yu et al. This is an open access article distributed under the Creative Commons Attribution License, which permits unrestricted use, distribution, and reproduction in any medium, provided the original work is properly cited.

The aim of this study was to evaluate ectopic in vivo bone formation with or without rat bone mesenchymal stem cells (rBMSCs) of an injectable Chitosan/Nanohydroxyapatite/Collagen (CS/nHAC) composite. The CS/nHAC composites were injected subcutaneously into the backs of Wistar rats with freshly loaded rBMSCs at a density of $10 \times 10^{6}$ cells/mL, and the CS/nHAC composites without cells were used as negative controls. New bone formation, degradation of composites, and degree of calcification were evaluated by Computed Tomography (CT) and three-dimensional (3D) CT reconstruction. Histological evaluations were performed to further assess bone structure and extracellular matrix by HE and Masson staining. The inflammatory reactions related to osteogenesis were also investigated in the present study. In comparison with the CS/nHAC composites, this study revealed that CS/nHAC/rBMSCs composites showed relatively higher percentage of calcification, better establishment of ECM, and less degradation rate. Meanwhile, different extents of inflammatory reactions were also observed in the CS/nHAC and $\mathrm{CS} / \mathrm{nHAC} / \mathrm{rBMSCs}$ explants at 2 and 4 weeks after implantation. Altogether, CS/nHAC/rBMSCs composites are superior to CS/nHAC composites in ectopic bone formation. In conclusion, the rBMSCs-seeded CS/nHAC composites may be beneficial to enhancing ectopic bone formation in vivo.

\section{Introduction}

In the recent years, significant progress has been made in the treatment of bone defect. With the development of bone tissue engineering, a variety of biomaterials appear to meet the challenges of inadequate supply of autograft [1], which is widely considered as the gold standard [2]. Bone graft substitutes have the advantages of unlimited availability, bearing no risk of disease transmission, and low incidence of complications [3]. As a promising candidate for bone tissue engineering, injectable biomaterials are popular for allowing the ability of noninvasive or minimally invasive implantation [4]. Besides, an ability to fill a desired shape and an easy incorporation with various therapeutic agents offer injectable biomaterials great potential in bone defect repair of any shape $[5,6]$.

It is universally acknowledged that an ideal biomaterial should be as close as possible to the natural ECM, which requires special care to the biocompatibility, biodegradability, and three-dimensional structure suitable for cell ingrowth and osteoinduction of the biomaterials [7-9]. Due to these limitations, very few composites turn out to 
meet these requirements. As a natural polymer obtained from chitin, chitosan has become a key component in bone tissue engineering in the last two decades [10]. Chitosan/nanohydroxyapatite composites have been shown to exhibit excellent biodegradability, biocompatibility, osteoconductivity, and even osteoinductivity [11, 12]. Renowned as a major organic matter of bone, collagen was combined with chitosan/nanohydroxyapatite to obtain a composite in our preliminary study. The feasibility of developing an injectable chitosan solution with excellent thermosensitivity in the presence of nHAC has been demonstrated. CS/nHAC makes the sol-gel transition when the solution is heated to $37^{\circ} \mathrm{C}$, which makes it form an aqueous solution with low viscosity to complete the injection, but it forms a gel at body temperature [13-15]. Our previous studies have shown that an injectable chitosan/nanohydroxyapatite/collagen composite exhibits features of natural bone in both main composition and microstructure, which can be expected as a candidate for systemic minimally invasive composites with osteogenic properties $[13,15]$.

The design of functionally active cells with composites to promote bone or cartilage formation is a character of bone tissue engineering [16]. Mesenchymal stem cells (MSCs) draw considerable attention because they can be easily isolated from a small aspirate of bone marrow and readily generate single-cell-derived colonies. MSCs have great potential in bone tissue engineering field. It has already been proved that MSCs improve the biocompatibility of CS/nHAC composites and enhance orthotopic bone formation in vivo [17].

In order to reduce the number of variables involved in bone formation, this time we employed a model of ectopic bone formation to perform our study. The goal of the current study was to evaluate the osteogenic ability of an injectable chitosan/nanohydroxyapatite/collagen composite with or without rat bone marrow mesenchymal stem cells (rBMSCs) during 4 weeks of subcutaneous implantation at the backs of rats.

\section{Materials and Methods}

2.1. Isolation and Cultivation of Rat Bone Marrow Mesenchymal Stem Cells. rBMSCs were isolated from bone marrow aspirates $(2 \mathrm{~mL})$, harvested by a standard procedure published previously $[18,19]$. To be specific, aspirates were obtained from the femurs of 8 eight-week-old male Wistar rats (Animal Research Center at Guangdong Province, China) under sterile conditions. Then, the marrow samples were flushed out with DMEM containing heparin $(100 \mu / \mathrm{mL})$ and became a cell pellet after being centrifuged at $1200 \mathrm{rpm}$ for $5 \mathrm{~min}$. Following removal of supernatant, cells were resuspended in Percoll gradient $(1.083 \mathrm{~g} / \mathrm{mL})$ and centrifuged at $1200 \times \mathrm{g}$ for $20 \mathrm{~min}$. After being washed twice with DMEM, the cells were seeded in low-glucose DMEM supplemented with FBS at a density of $5 \times 10^{6}$ cells $/ \mathrm{mL}$. After 2 days, the nonadherent cells were removed, while the adherent cells expanded under standard conditions $\left(5 \% \mathrm{CO}_{2}, 37^{\circ} \mathrm{C}\right)$ with the culture medium changed twice weekly. The third passage of cells at a confluence of approximately $80 \%-90 \%$ was used
TABLE 1: Major molecular components and microstructure of CS/nHAC as compared with natural bone. The molecular components, the mineralization of collagen fibrils, and the arrangement of mineralized collagen fibrils are the first, the second, and the third hierarchical levels in natural bone, respectively.

\begin{tabular}{|c|c|c|}
\hline & CS/nHAC & Natural bone \\
\hline $\begin{array}{l}\text { Major molecular } \\
\text { components }\end{array}$ & $\begin{array}{l}\text { HA, collagen, } \\
\text { phosphate, and } \\
\text { carbonates }\end{array}$ & $\begin{array}{l}\text { HA, water, collagen, } \\
\text { and other proteins }\end{array}$ \\
\hline $\begin{array}{l}\text { Mineralization of } \\
\text { collagen fibrils }\end{array}$ & $\begin{array}{l}\text { Small HA crystals } \\
\text { associated with } \\
\text { collagen fibrils } \\
\text { orienting their } c \text {-axis }\end{array}$ & $\begin{array}{l}\text { Plate-like HA crystals } \\
\text { associated with } \\
\text { collagen fibrils } \\
\text { orienting their } c \text {-axis }\end{array}$ \\
\hline $\begin{array}{l}\text { Arrangement of } \\
\text { the mineralized } \\
\text { collagen fibrils }\end{array}$ & $\begin{array}{l}\text { Associated as } \\
\text { bundles, aligned } \\
\text { along their long axis }\end{array}$ & $\begin{array}{l}\text { Associated as } \\
\text { bundles, often aligned } \\
\text { along their long axis }\end{array}$ \\
\hline
\end{tabular}

in our study. All of the procedures involving animals were performed in accordance with the Institutional Animal Care and Use Committee of Southern Medical University.

2.2. Preparation of $C S / n H A C$ and $C S / n H A C / r B M S C s$. The CS/nHAC composite was synthesized by a chemical modification route as described earlier $[13,15,17,20]$. Referring to our previous study, the main composition and the microstructure of $\mathrm{CS} / \mathrm{nHAC}$ are shown in Table 1 . It can be concluded that CS/nHAC is similar to natural bone in both molecular structure and microstructure [13]. For the CS/nHAC group, initially, nHAC powder was obtained by self-assembly of nanofibrils of mineralized collagen sterilized by $\gamma$-ray irradiation $(1.5 \mathrm{Mrad})$. Then, with the ratio of $1: 1 \mathrm{in}$ weight, nHAC powder was gently mixed with the chitosan solution, which was obtained by dissolving $2 \mathrm{~g}$ of chitosan in hydrochloric acid solution $(98 \mathrm{~mL}, 0.1 \mathrm{M})$. As for the resulting solution, droplets of $\beta$-glycerophosphate solution $(30 \%(\mathrm{w} / \mathrm{v}))$ were added to adjust the $\mathrm{pH}$ value to 7.0.

The CS/nHAC/rBMSCs composites were obtained by seeding rBMSCs into the CS/nHAC solution, prepared by the same procedure as in CS/nHAC group. Released from DMEM, the rBMSCs (passage 3) were suspended in lowglucose DMEM containing 10\% FBS. Then, the suspended cells were added to the CS/nHAC solution at a density of $10 \times 10^{6}$ cells $/ \mathrm{mL}$ and were gently mixed to achieve a uniform suspension; $10 \mathrm{nmol} / \mathrm{L}$ of dexamethasone, $50 \mathrm{mg} / \mathrm{L}$ of ascorbic acid, and $10 \mathrm{mmol} / \mathrm{L}$ of $\beta$-glycerophosphate were added to CS/nHAC/rBMSCs solution in order to promote cellular differentiation of rBMSCs. All procedures in both groups were carried out under sterile conditions.

2.3. Subcutaneous Implantation. Eight eight-week-old male Wistar rats weighing 250-300 g were used. The left side of the back (1.5 to $2 \mathrm{~cm}$ away from the midline) in each rat was chosen as the implantation area, while the right side (1.5 to $2 \mathrm{~cm}$ away from the midline) was untreated. Four rats were injected with CS/nHAC/rBMSCs subcutaneously, and the other four rats with CS/nHAC were considered as negative controls. No prophylactic medication was administered 


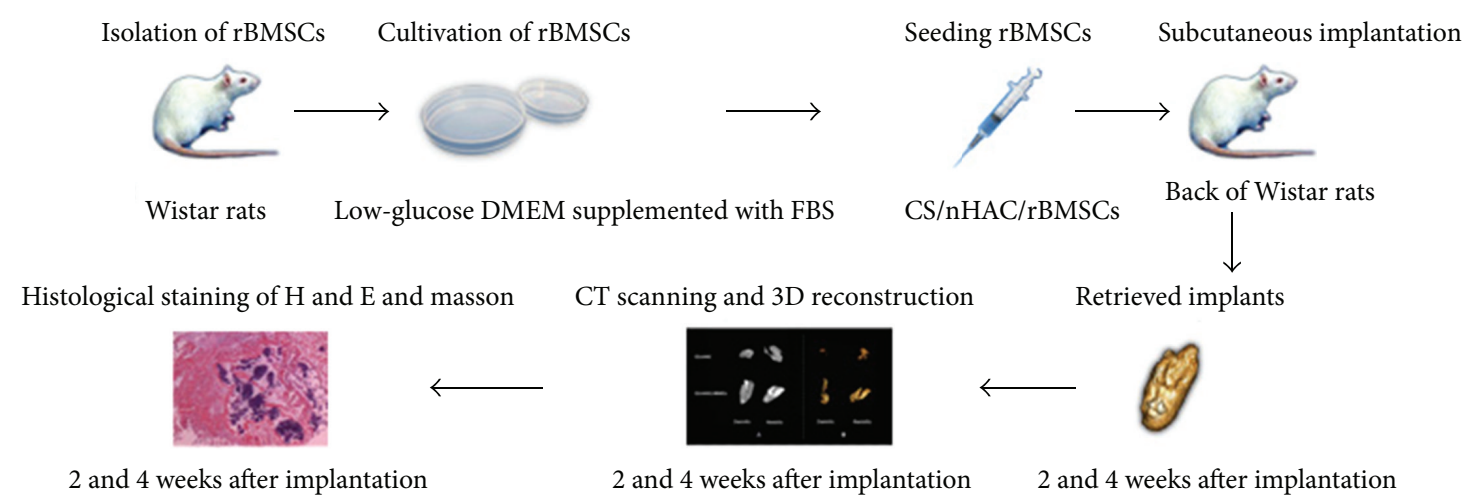

FIGURE 1: Schematic representation of experimental strategy: preparation of CS/nHAC/rBMSCs is demonstrated as an example.

before and after the implantation. The animals were allowed to walk about 24 hours after the implantation. After 2 and 4 weeks of implantation, the animals were sacrificed, and the implants were retrieved.

\subsection{Computed Tomography (CT) Scanning and Measurement} of Bone Density. Computed tomography (CT) scanning was performed to investigate new bone formation in the retrieved implants by using a Philips CT scanner (Phillips/Brilliance 64, Phillips, The Netherlands). At 2 and 4 weeks after implantation, the retrieved implants of both groups were scanned by $\mathrm{X}$-ray source energy with a resolution mode of $10 \mu \mathrm{m}(x / y / z)$ at parameters of $120 \mathrm{kV}, 150 \mathrm{~mA}$, and $0.5 \mathrm{~s}$ imaging time. Bone density measurement was performed on the foundation of determining the region of interest (ROI) in each slice of samples. ROI was defined by a senior pathologist using PHILIPS Brilliance Workspace Version 3.5 (Philips Medical Systems, USA) software. The data were obtained by taking the average of all of the CT values (HU) in ROI of three different slices in each sample. To provide an intuitive impression of the degradation properties and calcification of the implants, 3D bone formation was acquired by using a locally written software, which was based on the Feldkamp algorithm.

2.5. Histological Evaluation. After CT scanning, explants in both groups were embedded in paraffin for 24 hours and then sectioned. The $5 \mu \mathrm{m}$ thick sections were then stained by hematoxylin and eosin (HE) and Masson for evaluation of bone marrow architecture, lineage-specific cell types, and extracellular matrix (ECM). To quantify the degree of inflammation, a skilled pathologist engaged in calculating the number of inflammatory cells in sections of CS/nHAC and $\mathrm{CS} / \mathrm{nHAC} / \mathrm{rBMSC}$ groups, taking the average in each group. Phagocytes/macrophages, lymphocytes, and multinucleated giant cells are defined as inflammatory cells. ImagePro Plus (6.0.0260 version, Media Cybernetics, USA) was used for calculation.

2.6. Statistical Analysis. The bone density data and the number of inflammatory cells in the CS/nHAC and $\mathrm{CS} / \mathrm{nHAC} / \mathrm{rBMSC}$ groups were analyzed by using $t$-test.
IBM SPSS Statistics 19.0 software was used to assess the statistical differences intragroup and intergroup. Statistical significance was defined at a $P$ value $<0.05$.

\section{Result and Discussion}

Ectopic bone models are extremely useful for in vivo bone formation study. With unique advantages of eliminating the effect of bone-stimulating cytokines, bone-forming cells, endogenous stem cells, and potentially bone-stimulating mechanotransduction, ectopic bone formation has become a significant step for determining the osteogenic activities of various composites, especially for those achieving satisfactory results in orthotopic sites [21]. Subcutaneous implantation is one of the most common used models, regarding its convenience and favorable success rate. Scott et al. have concluded that bone marrow mesenchymal stem cells (BMSCs) are the most commonly studied cell types in subcutaneous models [21]. To determine the role of BMSCs in an injectable composite, we performed the ectopic bone model to evaluate the variability between the injectable CS/nHAC/rBMSCs and CS/nHAC composites. For an optimal outcome, we applied dexamethasone, ascorbic acid, and $\beta$-glycerophosphate to rBMSCs, which have been reported to promote more extensive bone formation in vivo [22, 23]. A subcutaneous bone model was established by injecting various composites into the left side of the back ( 1.5 to $2 \mathrm{~cm}$ away from the midline) in each rat. Computed tomography and histology were selected to assess the ectopic bone formation at 2 and 4 weeks after implantation (Figure 1).

3.1. CT Analysis of Implant Formation. Images of plain CT scanning provided evidence of calcification on the remaining composites (Figure 2(a)), which indicated the possibility of new bone formation. We were able to discover that calcification areas of both groups were just within the margin of remaining composites. It is obvious that the proportion of calcification area in CS/nHAC/rBMSCs was larger than that in CS/nHAC at 2 and 4 weeks after implantation, respectively, which demonstrated a more active osteogenesis site induced by $\mathrm{CS} / \mathrm{nHAC} / \mathrm{rBMSCs}$, in comparison with CS/nHAC. 


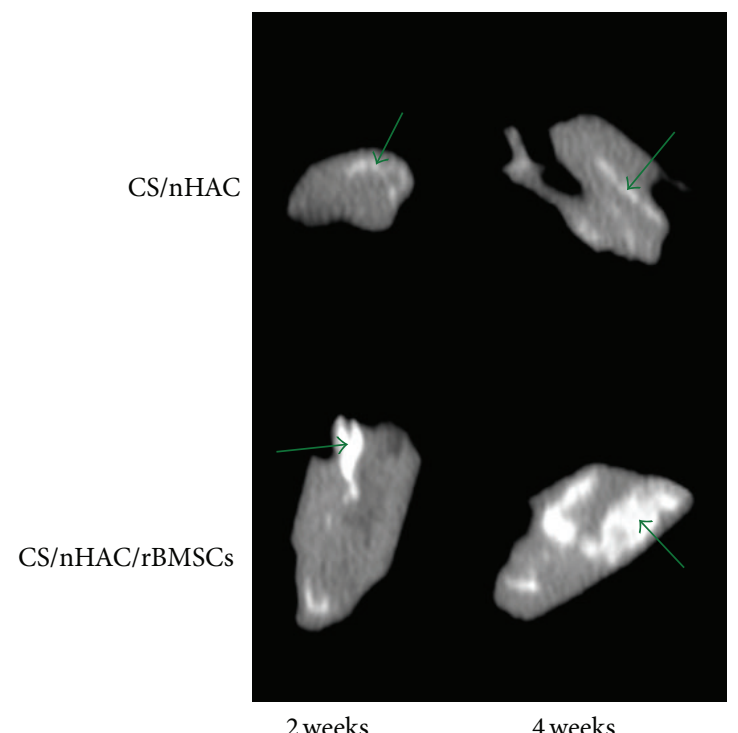

(a)

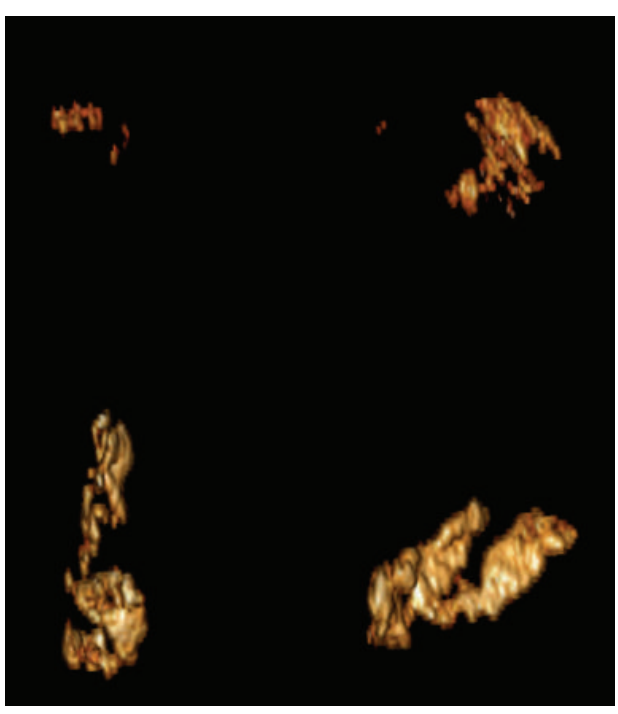

2 weeks

4 weeks

(b)

FIGURE 2: CT images of implant formation of CS/nHAC and CS/nHAC/rBMSCs at 2 and 4 weeks after implantation. (a) Images of plain CT scanning reveal calcification (green arrow). (b) Images of three-dimensional CT reconstruction demonstrate the remaining composites.

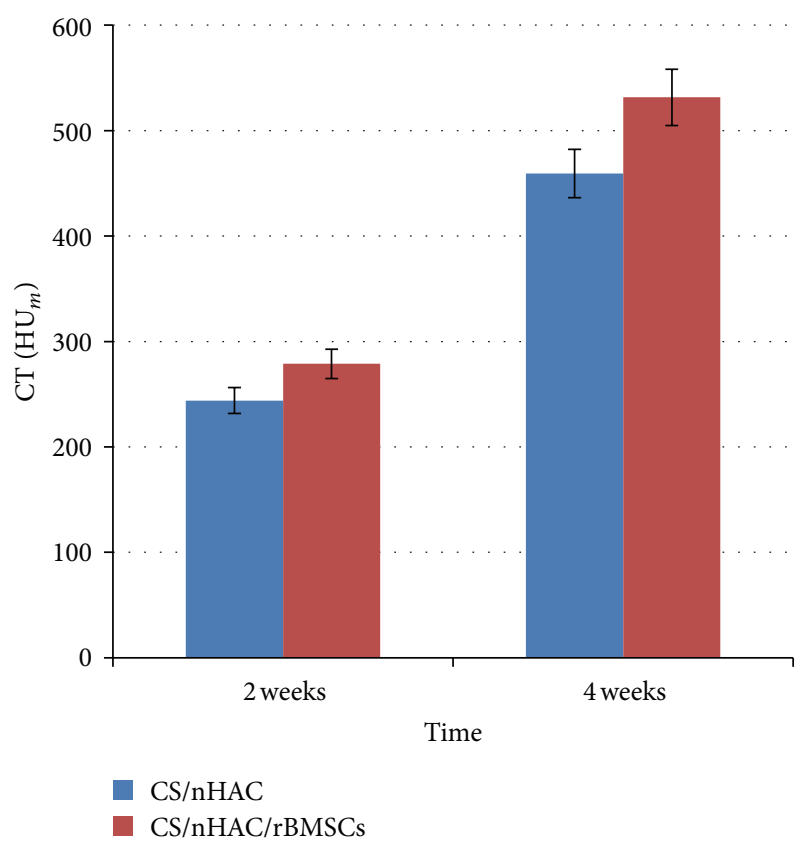

FIgURE 3: CT value $\left(\mathrm{HU}_{m}\right)$ of the implants in CS/nHAC and $\mathrm{CS} / \mathrm{nHAC} / \mathrm{rBMSCs}$ at 2 and 4 weeks after implantation. The data are presented as mean \pm standard deviation $\left(n=8 ;{ }^{*} P<0.05\right)$.

Three-dimensional CT reconstruction offers a convenience to visualize the implant formation. The images of the CS/nHAC/rBMSCs and CS/nHAC composites after implantation by $3 \mathrm{D}$ reconstruction taken at 2 and 4 weeks were obtained in this study (Figure 2(b)). It is universally acknowledged that degradation of composites plays an important role in new bone formation. On one hand, the persistence of composites within the implantation bed has been shown to restrict new bone formation. On the other hand, a fast degradation rate of composites is associated with failure in osteoconduction. An optimal degradation rate should be parallel to the speed of new bone formation [24]. In this study, a difference of degradation rates could be detected between the CS/nHAC/rBMSCs and CS/nHAC, with the volume of CS/nHAC remaining less at 2 and 4 weeks after implantation, as compared with CS/nHAC/rBMSCs. Another important finding was that the outlines of the remaining composites in both groups were extremely unclear, especially in CS/nHAC/rBMSCs, suggesting an ingrowth of surrounding tissue. Concerning the preferable osteogenic ability of $\mathrm{CS} / \mathrm{nHAC} / \mathrm{rBMSC}$ and its relatively slow degradation rate, as compared with CS/nHAC, further studies on the appropriate degradation rate could be carried out.

3.2. Computed Tomography Measurement of Bone Density. As a quantitative bone density measurement, the use of computed tomography has continued to grow in the field of bone implantation. Previous studies have shown the correlation between bone density values (the Hounsfield unit) and bone qualities $[25,26]$. Therefore, the Hounsfield unit $\left(\mathrm{HU}_{m}\right)$ was chosen for determination of bone density at 2 and 4 weeks after implantation in this study. The bone intensity values are shown in Figure 3. By loading rBMSCs with CS/nHAC, the CT intensity of CS/nHAC/rBMSCs was significantly higher than that of CS/nHAC at 2 and 4 weeks after implantation $(P<0.05)$. According to the mean $\mathrm{HU}$, it can be concluded that the increase of CT intensity was statistically significant $(P<0.05)$ in both cases within the duration of implantation up to 4 weeks (Figure 3). 


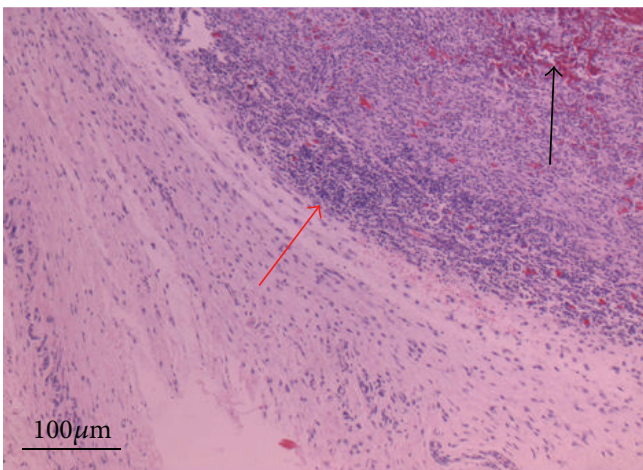

(a)

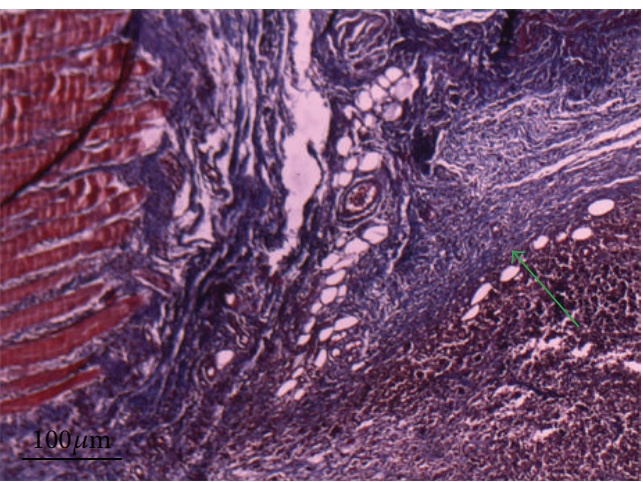

(c)

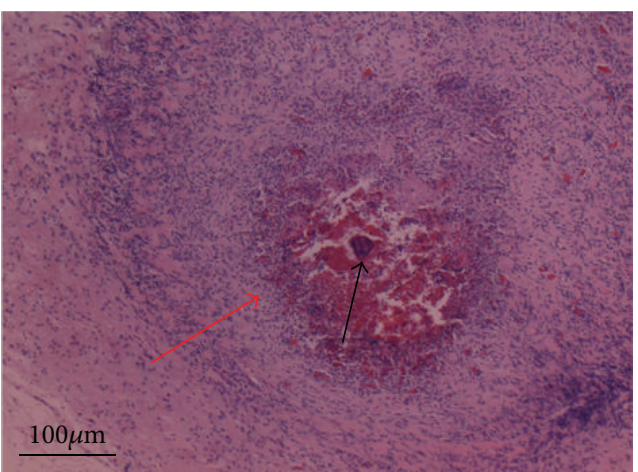

(b)

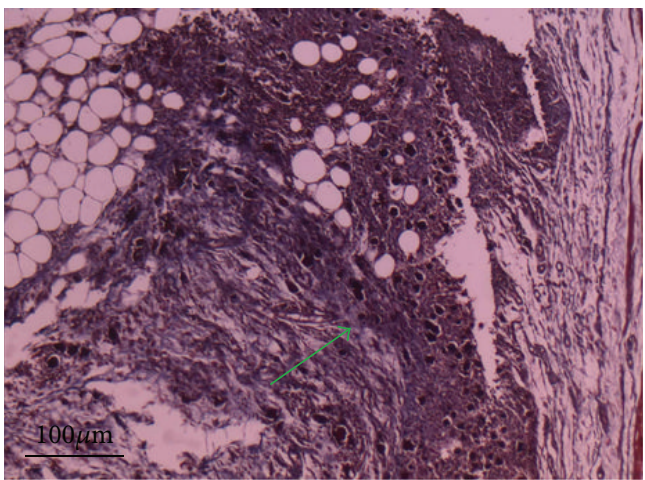

(d)

FIGURE 4: Light microscopic images of CS/nHAC induced bone formation at 2 and 4 weeks after implantation (microscopic magnification 100x) reveal weak evidence of bone formation with calcification (black arrow), collagen (green arrow), and inflammatory cells (red arrow): (a) HE, 2 weeks; (b) HE, 4 weeks; (c) Masson, 2 weeks; and (d) Masson, 4 weeks.

Furthermore, the mean CT intensity of both groups at 2 weeks indicated great possibility of calcification, especially in CS/nHAC/rBMSCs, corresponding to the de Oliveira R C $\mathrm{G}$ team's finding of bone intensity in CT values [26]. All of these results indicated the possibility of early osteogenesis in CS/nHAC/rBMSCs and CS/nHAC groups. As compared with $\mathrm{CS} / \mathrm{nHAC}, \mathrm{CS} / \mathrm{nHAC}$ seeded with $10 \times 10^{6}$ cells $/ \mathrm{mL}$ rBMSCs demonstrated greater possibility of bone formation.

3.3. Histological Analysis of Ectopic Bone Formation. In the current histological examination, percentage of calcification, inflammatory reactions related to implantation, and establishing of ECM were revealed by HE and Masson staining. For the CS/nHAC group, large amounts of inflammatory cells and fibrous tissue can be easily observed over the duration of 4 weeks after implantation, while a persistent low percentage of calcification was demonstrated (Figures 4(a) and 4(b)). Hardly any collagen can be seen by Masson staining at 2 weeks. A small amount of newly formed collagen bundles were sparsely and unevenly distributed in implant area at 4 weeks (Figures $4(\mathrm{c})$ and $4(\mathrm{~d})$ ), providing weak evidence of bone formation induced by CS/nHAC. In case of CS/nHAC/rBMSCs, the percentage of calcification significantly increased with time (Figures 5(a) and 5(b)), indicating an active bone formation site. Meanwhile, moderate amounts of inflammatory cells can be observed at 2 weeks after
TABLE 2: The number of inflammatory cells observed in CS/nHAC and CS/nHAC/rBMSCs at 2 and 4 weeks after implantation.

\begin{tabular}{lcc}
\hline & 2 weeks $\left(\right.$ cells $\left./ \mathrm{cm}^{2}\right)$ & 4 weeks $\left(\mathrm{cells} / \mathrm{cm}^{2}\right)$ \\
\hline $\mathrm{CS} / \mathrm{nHAC}$ & $37.6 \pm 1.5$ & $28.3 \pm 1.5$ \\
$\mathrm{CS} / \mathrm{nHAC} / \mathrm{rBMSCs}$ & $23.0 \pm 1.2$ & $12.0 \pm 1.5$ \\
\hline
\end{tabular}

implantation, and the cell number decreased sharply by 4 weeks. Numerous newly formed collagen bundles turned out to form homogeneously in the core of implants over the duration of 4 weeks (Figures 5(c) and 5(d)). Together, all of these histological findings demonstrated the possibility of new bone formation induced by CS/nHAC/rBMSCs, while few histological findings supported the osteogenic ability of CS/nHAC.

3.4. The Relationship between Osteogenesis and Inflammatory Reactions. Regarded as "foreign bodies", biomaterials are known to act on inflammatory reactions within the implantation bed. The inflammatory reaction is crucial for degradation of the biomaterials and vascularization of the implantation bed, thus contributing to new bone formation [27-29]. In our study, the relationship between osteogenesis and inflammatory reactions draws considerable attention. Inflammatory reactions observed in histological sections were measured by calculating the number of inflammatory 


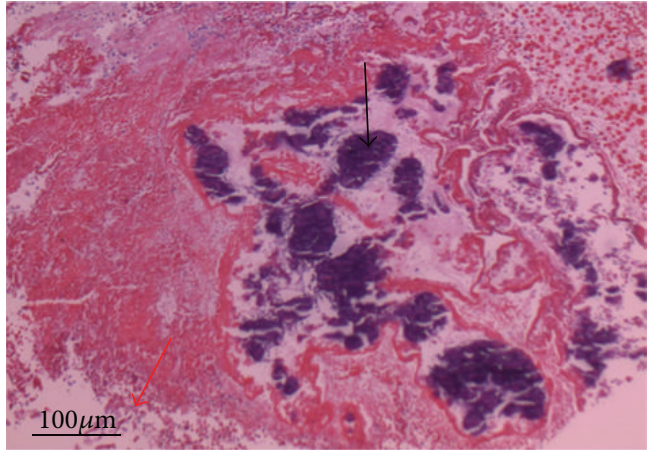

(a)

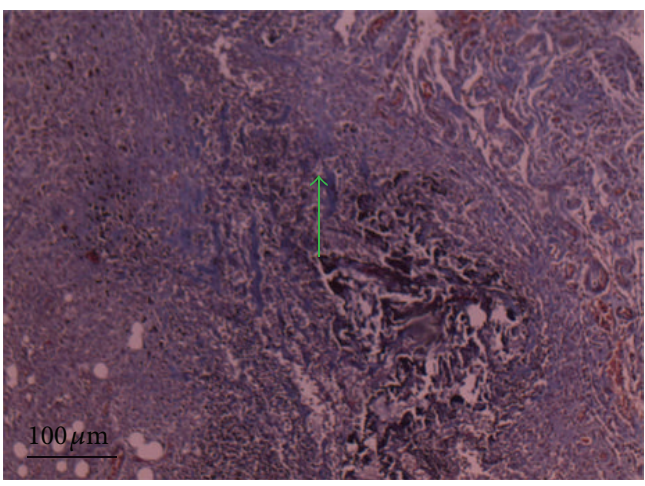

(c)

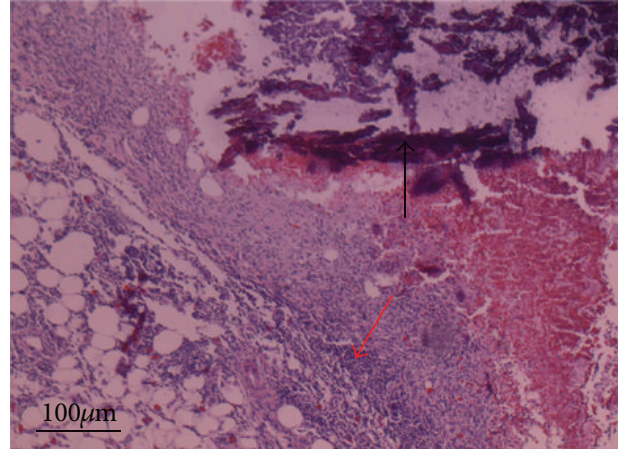

(b)

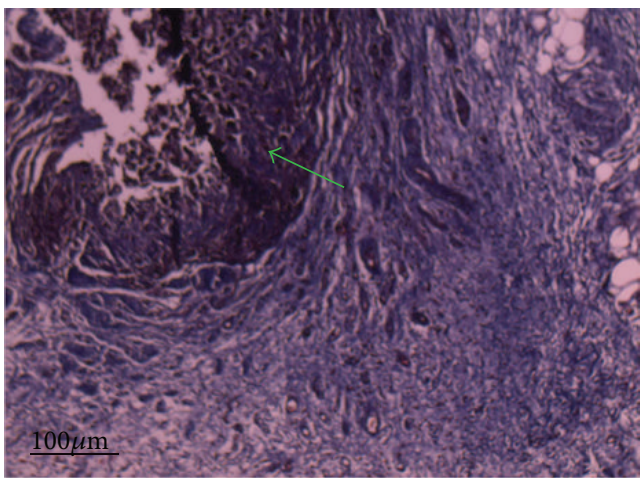

(d)

FIGURE 5: Light microscopic images of CS/nHAC/rBMSCs induced bone formation at 2 and 4 weeks after implantation (microscopic magnification 100x) indicate great potential of bone formation with calcification (black arrow), collagen (green arrow), and inflammatory cells (red arrow): (a) HE, 2 weeks; (b) HE, 4 weeks; (c) Masson, 2 weeks; and (d) Masson, 4 weeks.

cells (Table 2). A resolution of inflammatory reactions within 4 weeks of implantation in CS/nHAC/rBMSCs was observed. Moreover, the inflammatory reactions could be cataloged to a relatively moderate level at the early stage of implantation, indicating a preferable brief inflammation, while the CS/nHAC turned out to show no sign of bone formation with the degree of inflammatory reactions remaining at a high level up to 4 weeks (Figure 6). As Henson reported, the adhesion of macrophages and foreign body giant cells as well as other inflammatory cells has been shown to release mediators of degradation such as reactive oxygen intermediates (ROIs, oxygen-free radicals), degradative enzymes, and acids into this privileged zone between the cell membrane and composite surface such that immediate buffering or inhibition of these mediators is delayed or reduced [30, 31]. Therefore, inflammation is closely related to the biodegradation of composites, which determines the capability of new bone formation. This theory might illustrate the results of our study. The persistent inflammation fluctuating at a high level in CS/nHAC group mediated a fast biodegradation of composites, which resulted in a failure to support new bone formation, while the preferable brief inflammation of $\mathrm{CS} / \mathrm{nHAC} / \mathrm{rBMSC}$ resulted in an optimal degradation rate contributing to new bone formation. Our present study only provided statistics about inflammatory reactions at certain time points, and more dynamic monitoring and specific analysis of the inflammatory reactions need to be further investigated.

\section{Conclusions}

In conclusion, this study has demonstrated that an injectable $\mathrm{CS} / \mathrm{nHAC} / \mathrm{rBMSC}$ composite has great potential in enhancing ectopic bone formation. In comparison with $\mathrm{CS} / \mathrm{nHAC}$ composites, rBMSCs seeded at a density of $10 \times 10^{6}$ cells $/ \mathrm{mL}$ on CS/nHAC showed relatively higher percentage of calcification, better establishment of ECM, and less degradation rate. Therefore, the use of rBMSCs with CS/nHAC composite to promote ectopic bone formation deserves considerable attention.

\section{Conflict of Interests}

The authors declare that they have no financial or personal relationship with any people or any organization that may inappropriately influence their work, and there is no professional or commercial interest of any kind in all of the commercial entities mentioned in our paper.

\section{Authors' Contribution}

Bo Yu and Yichen Zhang contributed equally to this paper. 


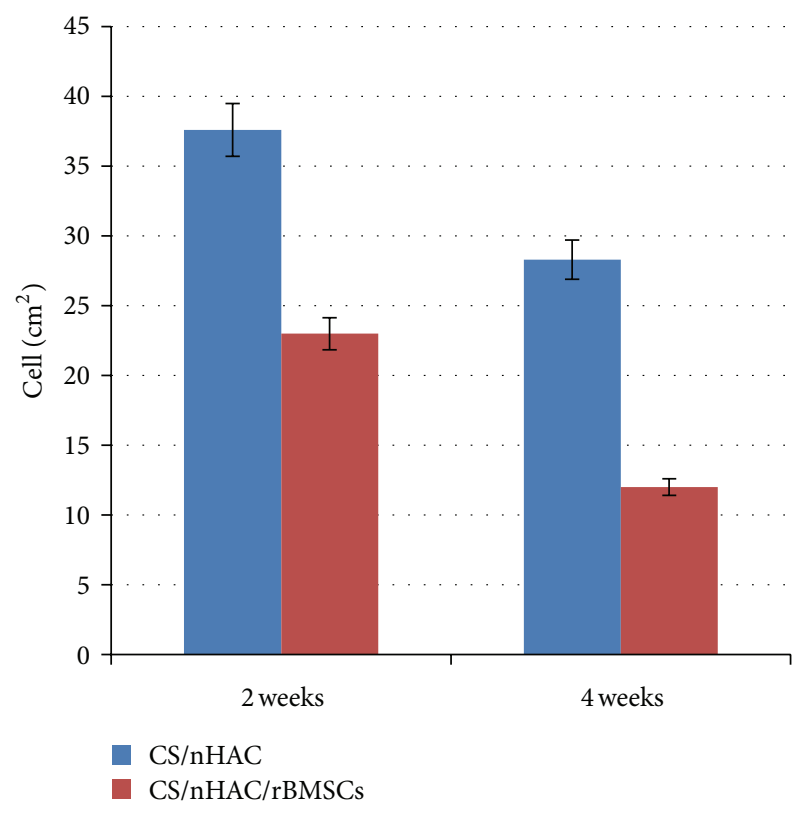

FIGURE 6: A comparison of inflammatory reactions measured by the number of inflammatory cells in both groups at the duration of implantation up to 4 weeks.

\section{Acknowledgments}

The authors are grateful for the financial support from the National Basic Research Program of China (973 Program no. 2011CB710901), the National Natural Science Foundation of China (no. 81101348 and no. 31000431), the Natural Science Foundation of Guangdong Province, China (no. 10451051501004727 and no. 10151051501000107), the Guangdong Medical Scientific Research Project (A2013385), Doctoral Fund of Ministry of Education, China (20114433120004), the Beijing Nova Program (no. 2010B011), the Program for New Century Excellent Talents (NCET) in University from Ministry of Education of China, Guangdong Province Undergraduate Innovative Experiment Program (no. 1212111013), the Shenzhen Strategic Emerging Industries Project (CXZZ20120618115626322), and the outstanding personal fund of Zhujiang Hospital.

\section{References}

[1] X. Li, Q. Feng, X. Liu, W. Dong, and F. Cui, “Collagen-based implants reinforced by chitin fibres in a goat shank bone defect model," Biomaterials, vol. 27, no. 9, pp. 1917-1923, 2006.

[2] R. Cancedda, P. Giannoni, and M. Mastrogiacomo, "A tissue engineering approach to bone repair in large animal models and in clinical practice," Biomaterials, vol. 28, no. 29, pp. 4240-4250, 2007.

[3] J. Venkatesan and S.-K. Kim, "Chitosan composites for bone tissue engineering-an overview," Marine Drugs, vol. 8, no. 8, pp. 2252-2266, 2010.

[4] M. B. Dreifke, N. A. Ebraheim, and A. C. Jayasuriya, "Investigation of potential injectable polymeric biomaterials for bone regeneration," Journal of Biomedical Materials Research A, vol. 101, no. 8, pp. 2436-2447, 2013.
[5] A. Gutowska, B. Jeong, and M. Jasionowski, "Injectable gels for tissue engineering," Anatomical Record, vol. 263, no. 4, pp. 342349, 2001.

[6] L. M. Boyd and A. J. Carter, "Injectable biomaterials and vertebral endplate treatment for repair and regeneration of the intervertebral disc," European Spine Journal, vol. 15, supplement 3, pp. S414-S421, 2006.

[7] F. M. Tonelli, A. K. Santos, K. N. Gomes et al., "Carbon nanotube interaction with extracellular matrix proteins producing scaffolds for tissue engineering," International Journal of Nanomedicine, vol. 7, pp. 4511-4529, 2012.

[8] X. Li, L. Wang, Y. Fan, Q. Feng, F. Z. Cui, and F. Watari, "Nanostructured scaffolds for bone tissue engineering," Journal of Biomedical Materials Research A, vol. 101, no. 8, pp. 24242435, 2013.

[9] X. Li, Q. Feng, W. Wang, and F. Cui, "Chemical characteristics and cytocompatibility of collagen-based scaffold reinforced by chitin fibers for bone tissue engineering," Journal of Biomedical Materials Research B, vol. 77, no. 2, pp. 219-226, 2006.

[10] M. Wang, "Developing bioactive composite materials for tissue replacement," Biomaterials, vol. 24, no. 13, pp. 2133-2151, 2003.

[11] Z. Li, L. Yubao, Y. Aiping, P. Xuelin, W. Xuejiang, and Z. Xiang, "Preparation and in vitro investigation of chitosan/nanohydroxyapatite composite used as bone substitute materials," Journal of Materials Science, vol. 16, no. 3, pp. 213-219, 2005.

[12] B. M. Chesnutt, Y. Yuan, K. Buddington, W. O. Haggard, and J. D. Bumgardner, "Composite chitosan/nano-hydroxyapatite scaffolds induce osteocalcin production by osteoblasts in vitro and support bone formation in vivo," Tissue engineering A, vol. 15, no. 9, pp. 2571-2579, 2009.

[13] Z. Huang, Q. Feng, B. Yu, and S. Li, "Biomimetic properties of an injectable chitosan/nano-hydroxyapatite/collagen composite," Materials Science and Engineering C, vol. 31, no. 3, pp. 683-687, 2011.

[14] X. Li, H. Liu, X. Niu et al., "The use of carbon nanotubes to induce osteogenic differentiation of human adipose-derived MSCs in vitro and ectopic bone formation in vivo," Biomaterials, vol. 33, no. 19, pp. 4818-4827, 2012.

[15] Z. Huang, J. Tian, B. Yu, Y. Xu, and Q. Feng, "A bone-like nanohydroxyapatite/collagen loaded injectable scaffold," Biomedical Materials, vol. 4, no. 5, Article ID 055005, 2009.

[16] Y. Xiao, S. Mareddy, and R. Crawford, "Clonal characterization of bone marrow derived stem cells and their application for bone regeneration," International Journal of Oral Science, vol. 2, no. 3, pp. 127-135, 2010.

[17] Z. Huang, Y. Chen, Q.-L. Feng et al., "In vivo bone regeneration with injectable chitosan/hydroxyapatite/collagen composites and mesenchymal stem cells," Frontiers of Materials Science, vol. 5, no. 3, pp. 301-310, 2011.

[18] J. Y. Sun, S. P. Ki, S. K. Moon, J. M. Rhee, G. Khang, and B. L. Hai, "Repair of diaphyseal bone defects with calcitriol-loaded PLGA scaffolds and marrow stromal cells," Tissue Engineering, vol. 13, no. 5, pp. 1125-1133, 2007.

[19] P. Kasten, J. Vogel, F. Geiger, P. Niemeyer, R. Luginbühl, and K. Szalay, "The effect of platelet-rich plasma on healing in criticalsize long-bone defects," Biomaterials, vol. 29, no. 29, pp. 39833992, 2008.

[20] Z. Huang, B. Yu, Q. Feng, S. Li, Y. Chen, and L. Luo, "In situ-forming chitosan/nano-hydroxyapatite/collagen gel for the delivery of bone marrow mesenchymal stem cells," Carbohydrate Polymers, vol. 85, no. 1, pp. 261-267, 2011. 
[21] M. A. Scott, B. Levi, A. Askarinam et al., "Brief review of models of ectopic bone formation," Stem Cells and Development, vol. 21, no. 5, pp. 655-667, 2012.

[22] A. Muraglia, I. Martin, R. Cancedda, and R. Quarto, "A nude mouse model for human bone formation in unloaded conditions," Bone, vol. 22, supplement 5, pp. 131S-134S, 1998.

[23] P. H. Krebsbach, S. A. Kuznetsov, K. Satomura, R. V. B. Emmons, D. W. Rowe, and P. G. Robey, "Bone formation in vivo: comparison of osteogenesis by transplanted mouse and human marrow stromal fibroblasts," Transplantation, vol. 63, no. 8, pp. 1059-1069, 1997.

[24] R. Langer and J. P. Vacanti, “Tissue engineering," Science, vol. 260, no. 5110, pp. 920-926, 1993.

[25] M. R. Norton and C. Gamble, "Bone classification: an objective scale of bone density using the computerized tomography scan," Clinical Oral Implants Research, vol. 12, no. 1, pp. 79-84, 2001.

[26] R. C. G. de Oliveira, C. R. Leles, L. M. Normanha, C. Lindh, and R. F. Ribeiro-Rotta, "Assessments of trabecular bone density at implant sites on CT images," Oral Surgery, Oral Medicine, Oral Pathology, Oral Radiology and Endodontology, vol. 105, no. 2, pp. 231-238, 2008.

[27] J. M. Anderson, A. Rodriguez, and D. T. Chang, "Foreign body reaction to biomaterials," Seminars in Immunology, vol. 20, no. 2, pp. 86-100, 2008.

[28] J. M. Anderson and A. K. McNally, "Biocompatibility of implants: lymphocyte/macrophage interactions," Seminars in Immunopathology, vol. 33, no. 3, pp. 221-233, 2011.

[29] E. Alsberg, H. J. Kong, Y. Hirano, M. K. Smith, A. Albeiruti, and D. J. Mooney, "Regulating bone formation via controlled scaffold degradation," Journal of Dental Research, vol. 82, no. 11, pp. 903-908, 2003.

[30] P. M. Henson, "The immunologic release of constituents from neutrophil leukocytes. I. The role of antibody and complement on nonphagocytosable surfaces or phagocytosable particles," Journal of Immunology, vol. 107, no. 6, pp. 1535-1546, 1971.

[31] P. M. Henson, "The immunologic release of constituents from neutrophil leukocytes. II. Mechanisms of release during phagocytosis, and adherence to nonphagocytosable surfaces," Journal of Immunology, vol. 107, no. 6, pp. 1547-1557, 1971. 

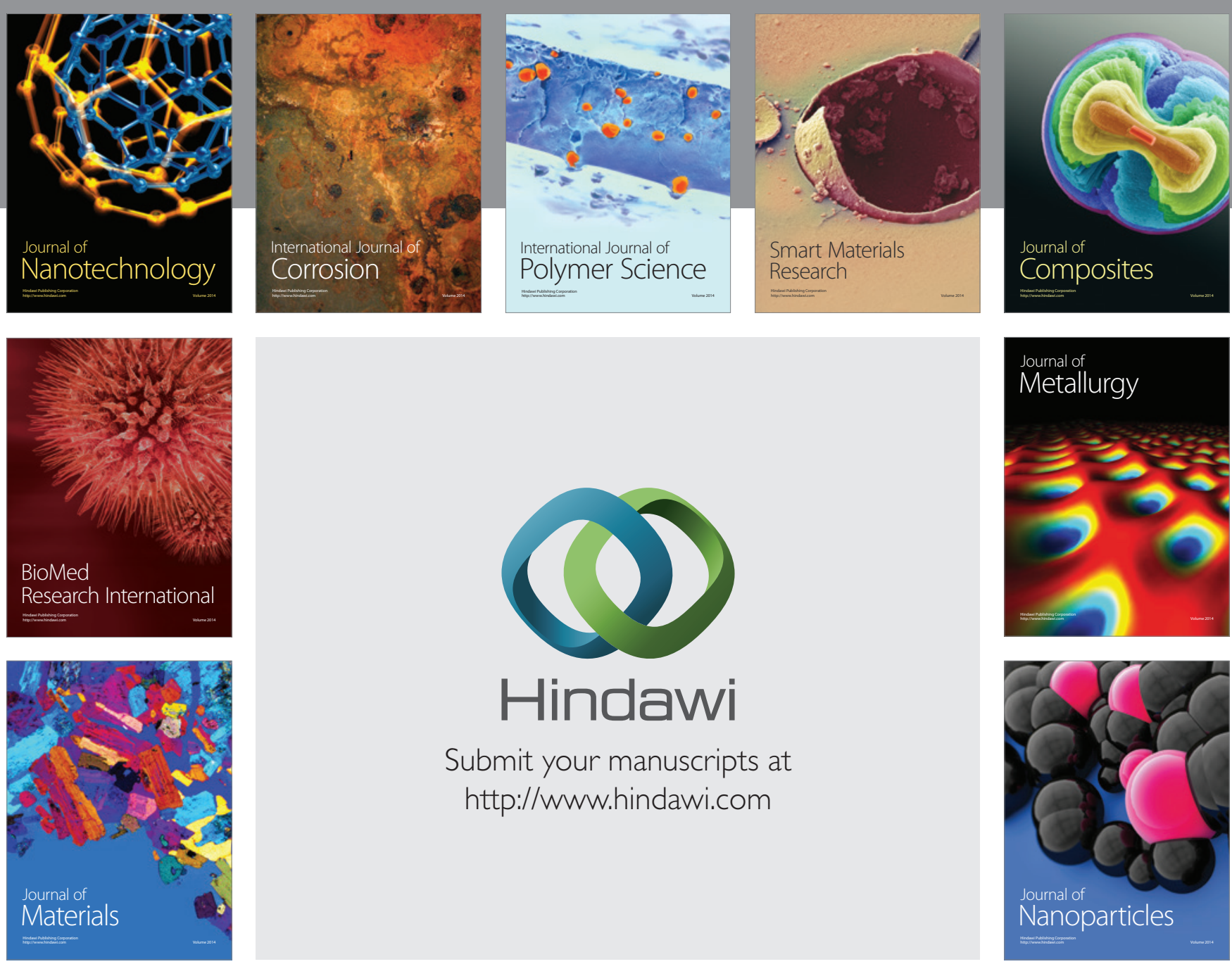

Submit your manuscripts at http://www.hindawi.com
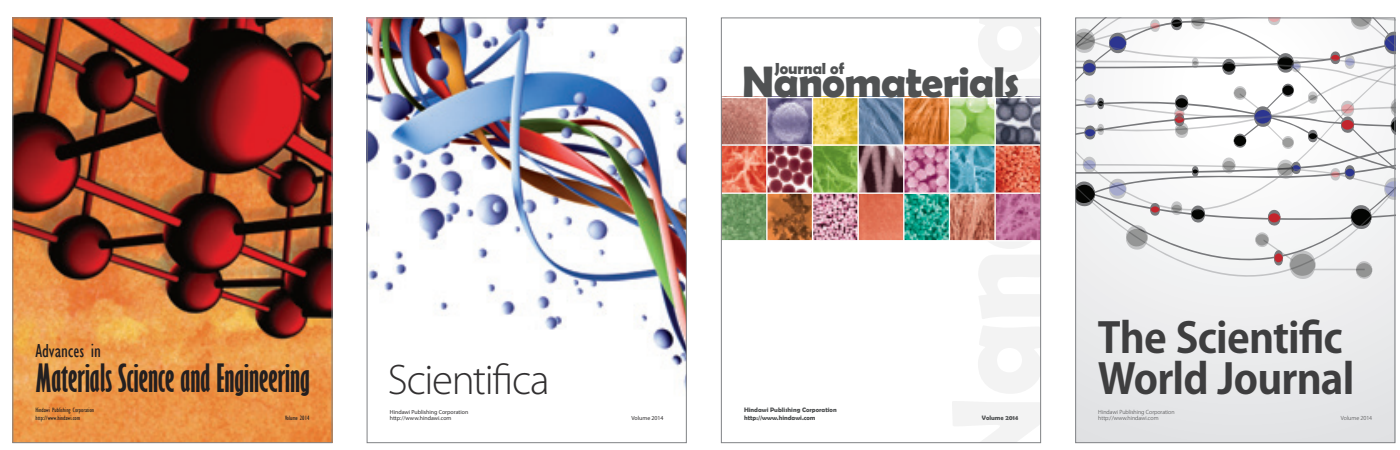

\section{The Scientific World Journal}
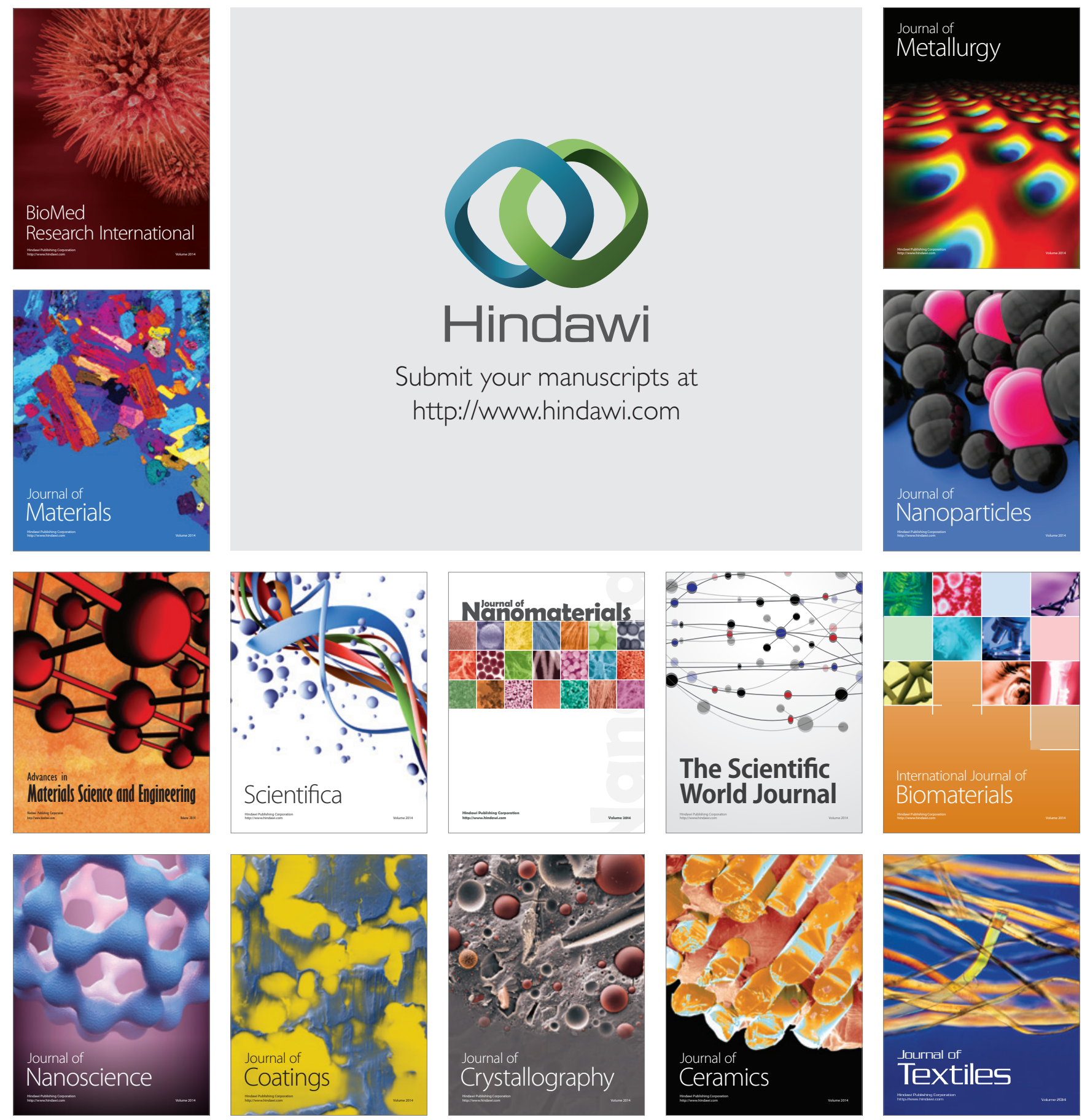